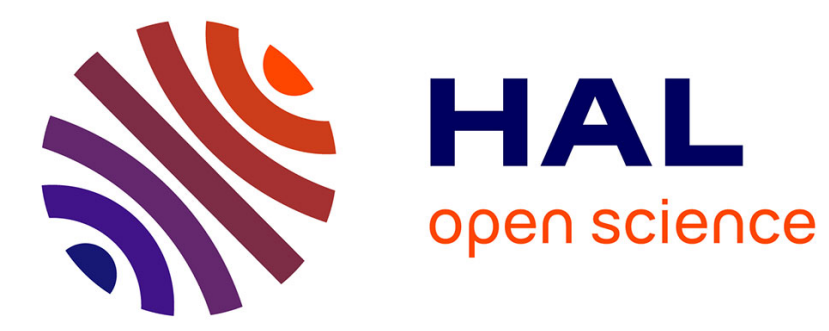

\title{
On robust stability of sine-Gordon equation
}

Denis Efimov, Emilia Fridman, Jean-Pierre Richard

\section{To cite this version:}

Denis Efimov, Emilia Fridman, Jean-Pierre Richard. On robust stability of sine-Gordon equation.

Proc. IEEE CDC, Dec 2019, Nice, France. hal-02418533

\section{HAL Id: hal-02418533 \\ https://hal.inria.fr/hal-02418533}

Submitted on 18 Dec 2019

HAL is a multi-disciplinary open access archive for the deposit and dissemination of scientific research documents, whether they are published or not. The documents may come from teaching and research institutions in France or abroad, or from public or private research centers.
L'archive ouverte pluridisciplinaire HAL, est destinée au dépôt et à la diffusion de documents scientifiques de niveau recherche, publiés ou non, émanant des établissements d'enseignement et de recherche français ou étrangers, des laboratoires publics ou privés. 


\title{
On robust stability of sine-Gordon equation
}

\author{
Denis Efimov, Emilia Fridman, Jean-Pierre Richard
}

\begin{abstract}
A perturbed sine-Gordon equation is considered under the restrictions on the model parameters corresponding to the single equilibrium in the noise-free case. First, a strict Lyapunov function is proposed for this dynamics and the conditions of strict passivity with a corresponding output are given. Second, the input-to-state stability property is investigated. The obtained theoretical results are illustrated by some simulations.
\end{abstract}

\section{INTRODUCTION}

There are many physical, biological and social systems, whose dynamics can be described by partial differential equations [3], [9], [14], [17]. The problems of stability analysis, estimator and controller design have been considered by many authors (see e.g. [12], [18], [16], [2]). Despite a significant advance in the analysis and synthesis of linear infinite-dimensional systems achieved the last decades, each nonlinear (partial) differential equation requires a particular attention and a separate theoretical development. Indeed, as for the conventional ordinary differential equations, in order to investigate and quantify a stability property for a nonlinear system, it is necessary to find a Lyapunov function, whose choice in the nonlinear case is always complicated and depends on the system dynamics.

This work is devoted to stability analysis of a damped sine-Gordon equation [6], [20], [17], [11], [15], [7], [8], which belongs to the class of hyperbolic distributed parameter systems with a globally Lipschitz nonlinearity, and which is one of the simplest models having soliton solutions. We will consider a particular case with one input applied on a boundary and another in the right-hand side of the partial differential equation. Under imposed restrictions, this nonlinear distributed parameter system in the absence of the inputs admits the only steady state at the origin. First, a kind of strict passivity conditions are established with the corresponding storage function, whose derivative along the system is strictly negative with zero input, and which can be considered as a Lyapunov function for the scenario without the input. This Lyapunov function recovers global asymptotic

D. Efimov is with Inria, Univ. Lille, CNRS, UMR 9189 - CRIStAL, F59000 Lille, France and with Department of Control Systems and Informatics, University ITMO, 197101 Saint Petersburg, Russia.

E. Fridman is with School of Electrical Engineering, Tel-Aviv University, Tel-Aviv 69978, Israel.

J.-P. Richard is with Centrale Lille, Univ. Lille, CNRS, UMR 9189 CRIStAL, F-59000 Lille, France.

This work was partially supported by the Ministry of Science and Higher Education of Russian Federation (passport of goszadanie no. 2019-0898) and by Israel Science Foundation (grant No 1128/14). The input-to-state stability conditions presented in Section $\mathrm{V}$ were developed under support of RSF (grant 17-19-01422) in ITMO University. stability conditions that were proved in [20] by using LaSalle invariance principle. Second, the conditions of global inputto-state stability are derived. To the best of the authors knowledge, it is the first time when such results are obtained, and a probable reason consists in non-quadratic terms of the suggested Lyapunov functions.

The paper is organized as follows. Preliminaries are given in Section II. The problem statement is presented in Section III with the main results in Section IV. Robustness with respect to external inputs is investigated in Section V. The results of simulation are given in Section VI. The final remarks and discussion are presented in Section VII.

\section{PRELIMINARIES}

The real numbers are denoted by $\mathbb{R}, \mathbb{R}_{+}=\{\tau \in \mathbb{R}: \tau \geq$ $0\}$. Euclidean norm for a vector $x \in \mathbb{R}^{n}$ will be denoted as $|x|$.

If $X$ is a normed space with norm $\|\cdot\|_{X}, \Omega \subset \mathbb{R}^{n}$ for some $n \geq 1$ and $\phi: \Omega \rightarrow X$, define

$$
\begin{gathered}
\|\phi\|_{L^{2}(\Omega, X)}^{2}=\int_{\Omega}\|\phi(s)\|_{X}^{2} d s, \\
\|\phi\|_{L^{\infty}(\Omega, X)}=e s s \sup _{s \in \Omega}\|\phi(s)\|_{X} .
\end{gathered}
$$

By $L^{\infty}(\Omega, X)$ and $L^{2}(\Omega, X)$ denote the spaces of functions $\Omega \rightarrow X$ with the properties $\|\cdot\|_{L^{\infty}(\Omega, X)}<+\infty$ and $\|\cdot\|_{L^{2}(\Omega, X)}<+\infty$, respectively. Let $C^{k}(\Omega, X)$ be the set of functions having continuous derivatives at least up to order $k \geq 0$ on $\Omega$. Denote by $H^{q}(\Omega, \mathbb{R})$ with $q \geq 0$ the Sobolev space of functions with derivatives through order $q$ in $L^{2}(\Omega, \mathbb{R})$.

\section{A. Input-to-state stability}

The theory of input-to-state stability (ISS) for infinite dimensional systems is presented in this subsection following [4], [13].

The triple $\Sigma=(X, U, \phi)$, consisting of the state space $X$, the space of admissible inputs $U$ (a normed function space) and the transition map $\phi: \mathbb{R}_{+} \times X \times U \rightarrow X$ is called a dynamical system if the following properties are satisfied:

- $\phi\left(0, x_{0}, u\right)=x_{0}$ for all $x_{0} \in X$ and all $u \in U$, and there is $T_{x_{0}, u}>0$ such that $\phi\left(t, x_{0}, u\right)$ exists for all $t \in\left[0, T_{x_{0}, u}\right)$;

- $\phi\left(t, x_{0}, u_{1}\right)=\phi\left(t, x_{0}, u_{2}\right)$ for any $t \in \mathbb{R}_{+}$, any $x_{0} \in X$ and all $u_{1}, u_{2} \in U$ such that $u_{1}(s)=u_{2}(s)$ for all $s \in[0, t]$

- for each $x_{0} \in X$ and each $u \in U$ the map $t \rightarrow$ $\phi\left(t, x_{0}, u\right)$ is continuous; 
- for all $x_{0} \in X$, all $u \in U$ and any $0 \leq t_{1} \leq t_{2}<T_{x_{0}, u}$ it holds $\phi\left(t_{2}, x_{0}, u\right)=\phi\left(t_{2}-t_{1}, \phi\left(t_{1}, x_{0}, u\right), u\right)$.

Thus, $\phi\left(T, x_{0}, u\right)$ denotes the state of a system $\Sigma$ at the time instant $0 \leq T<T_{x_{0}, u}$ if its initial state (at the instant 0 ) was $x_{0} \in X$ and the input $u \in U$ was applied for all $t \in[0, T)$. It is assumed that if $T_{x_{0}, u}<+\infty$, then for any $\epsilon>0$ there exists $t \in\left[0, T_{x_{0}, u}\right)$ such that $\left\|\phi\left(t, x_{0}, u\right)\right\|_{X}>\epsilon$ (the socalled "boundedness-implies-continuation" property). Next, it is assumed that for any $u \in U$ and $s \geq 0$, a function $\tilde{u}$ defined by $\tilde{u}(t)=u(t+s)$ for all $t \geq 0$ also belongs to $U$ and $\|\tilde{u}\|_{U} \leq\|u\|_{U}$; if $u_{1}, u_{2} \in U$, then for any $s>0$ the input $u \in U$ with $u(t)=\left\{\begin{array}{ll}u_{1}(t) & t \in[0, s] \\ u_{2}(t) & t>s\end{array} ;\right.$ and $0 \in U$.

An element of the state space $x_{e} \in X$ is called an equilibrium point of $\Sigma$ if $\phi\left(t, x_{e}, 0\right)=x_{e}$ for all $t \geq 0$, and for further analysis we will assume that $x_{e}=0(0 \in X)$.

A continuous function $\sigma: \mathbb{R}_{+} \rightarrow \mathbb{R}_{+}$belongs to class $\mathcal{K}$ if it is strictly increasing and $\sigma(0)=0$; it belongs to class $\mathcal{K}_{\infty}$ if it is also radially unbounded. A continuous function $\beta: \mathbb{R}_{+} \times \mathbb{R}_{+} \rightarrow \mathbb{R}_{+}$belongs to class $\mathcal{K} \mathcal{L}$ if $\beta(\cdot, r) \in \mathcal{K}$ and $\beta(r, \cdot)$ is a strictly decreasing to zero for any fixed $r \in \mathbb{R}_{+}$.

Definition 1. A dynamical system $\Sigma$ is called locally ISS, if there exist $\rho_{X}>0, \rho_{U}>0, \varpi \in \mathcal{K} \mathcal{L}$ and $\gamma \in \mathcal{K}$ such that the inequality

$$
\left\|\phi\left(t, x_{0}, u\right)\right\|_{X} \leq \varpi\left(\left\|x_{0}\right\|_{X}, t\right)+\gamma\left(\|u\|_{U}\right)
$$

is satisfied for all $t \geq 0$ and for all $x_{0} \in X, u \in U$ provided that $\left\|x_{0}\right\|_{X} \leq \rho_{X}$ and $\|u\|_{U} \leq \rho_{U}$.

This system $\Sigma$ is called ISS if $\rho_{X}=\rho_{U}=+\infty$.

The most common choices are $X=L^{2}\left(\mathbb{R}_{+}, \mathbb{R}^{n}\right)$ and $U=$ $L^{\infty}\left(\mathbb{R}_{+}, \mathbb{R}^{m}\right)$ or $U=L^{2}\left(\mathbb{R}_{+}, \mathbb{R}^{m}\right)$ for some natural numbers $n$ and $m$ [5].

Definition 2. A function $V \in C^{0}\left(X, \mathbb{R}_{+}\right)$is called a local ISS-Lyapunov function for $\Sigma$, if there exist $\rho_{X}>0, \rho_{U}>0$, $\alpha_{1}, \alpha_{2} \in \mathcal{K}_{\infty}, \chi \in \mathcal{K}$ and a positive definite function $\varsigma \in$ $C^{0}\left(\mathbb{R}_{+}, \mathbb{R}_{+}\right)$such that

$$
\begin{gathered}
\alpha_{1}\left(\|x\|_{X}\right) \leq V(x) \leq \alpha_{2}\left(\|x\|_{X}\right), \\
\|x\|_{X} \geq \chi\left(\|u\|_{U}\right) \Rightarrow \dot{V}(x, u) \leq-\varsigma\left(\|x\|_{X}\right)
\end{gathered}
$$

for all $x \in X, u \in U$ provided that $\left\|x_{0}\right\|_{X} \leq \rho_{X}$ and $\|u\|_{U} \leq \rho_{U}$, where

$$
\dot{V}(x, u)=\varlimsup_{h \rightarrow 0} \frac{V(\phi(h, x, u))-V(x)}{h} .
$$

A function $V \in C^{0}\left(X, \mathbb{R}_{+}\right)$is called an ISS-Lyapunov function for $\Sigma$, if the above properties hold for $\rho_{X}=\rho_{U}=$ $+\infty$.

Theorem 1. [4] If a dynamical system $\Sigma$ possesses a (local) ISS-Lyapunov function, then it is (locally) ISS.

In [13] it has been additionally shown that for a Lipschitz dynamical system $\Sigma$, the existence of a local ISS-Lyapunov function is necessary and sufficient for the ISS property.

\section{B. Useful relations}

Denote $I=[0, \ell]$ for some $\ell>0$.

Lemma 1. [10] Wirtinger's Inequality. Let $z \in H^{1}(I, \mathbb{R})$, then

$$
\int_{0}^{\ell} z^{2}(x) d x \leq \frac{b \ell^{2}}{\pi^{2}} \int_{0}^{\ell}\left(\frac{d z(x)}{d x}\right)^{2} d x
$$

with $b=1$ provided that $z(0)=z(\ell)=0$; if only $z(0)=0$ or $z(\ell)=0$, then $b=4$.

For a differentiable function $z: I \rightarrow \mathbb{R}$ define the shorthand notation:

$$
z_{x}(x)=\frac{\partial z}{\partial x}(x), \quad z_{x x}(x)=\frac{\partial^{2} z}{\partial x^{2}}(x),
$$

and if the origin of the argument $x$ is clear from the context or unimportant we will write $z_{x}$ and $z_{x x}$ (similarly for a function of two arguments $z(t, x)$ with $z_{t}$ or $\left.z_{x t}\right)$.

Proposition 1. The following equalities are satisfied for any $z \in H^{1}\left(\mathbb{R}_{+} \times I, \mathbb{R}\right)$ for all $t \in \mathbb{R}_{+}$and $x \in I$ (i.e. $z(t, x)$ ):

$$
\begin{gathered}
\int_{0}^{\ell} z_{t} z_{x x} d x=z_{t}(t, \ell) z_{x}(t, \ell)-z_{t}(t, 0) z_{x}(t, 0)-\int_{0}^{\ell} z_{t x} z_{x} d x \\
\int_{0}^{\ell} z_{x x} \sin z d x=\sin z(t, \ell) z_{x}(t, \ell)-\sin z(t, 0) z_{x}(t, 0) \\
\quad-\int_{0}^{\ell} z_{x}^{2} \cos z d x \\
\int_{0}^{\ell} z z_{x x} d x=z(t, \ell) z_{x}(t, \ell)-z(t, 0) z_{x}(t, 0)-\int_{0}^{\ell} z_{x}^{2} d x
\end{gathered}
$$

Proof. All these relations are obtained using the integration by parts.

\section{PROBlem STATEMENT}

Consider a damped one-dimensional sine-Gordon partial differential equation:

$$
\begin{gathered}
z_{t t}(t, x)=-\alpha z_{t}(t, x)+k z_{x x}(t, x)+\beta \sin z(t, x) \\
+u_{1}(t), t \in \mathbb{R}_{+}, x \in I \\
z(0, x)=z^{0}(x), z_{t}(0, x)=z^{1}(x), x \in I \\
z(t, 0)=0, z_{x}(t, \ell)=u_{2}(t), t \in \mathbb{R}_{+},
\end{gathered}
$$

where $z(t, x) \in \mathbb{R}$ and $\zeta(t, \cdot)=\left[\begin{array}{c}z(t, \cdot) \\ z_{t}(t, \cdot)\end{array}\right] \in \mathcal{Z}(I, \mathbb{R})=$ $H_{0}^{1}(I, \mathbb{R}) \times L^{2}(I, \mathbb{R})$ is the state function, where

$$
H_{0}^{1}(I, \mathbb{R})=\left\{z \in H^{1}(I, \mathbb{R}): z(0)=0\right\}
$$

and denote

$$
\|\zeta\|_{\mathcal{Z}(I, \mathbb{R})}=\left\|z_{x}\right\|_{L^{2}(I, \mathbb{R})}+\left\|z_{t}\right\|_{L^{2}(I, \mathbb{R})}
$$

$u(t) \in \mathbb{R}^{2}$ is the input signal, $u \in L^{\infty}\left(\mathbb{R}_{+}, \mathbb{R}^{2}\right) ; \alpha>0$, $k>0$ and $\beta \in \mathbb{R}$ are constant parameters; $z^{0} \in H_{0}^{1}(I, \mathbb{R})$ and $z^{1} \in L^{2}(I, \mathbb{R})$ are given initial condition functions satisfying the boundary constraints; $I=[0, \ell]$ for some $\ell>0$. The dynamics of (1) is globally well posed [17], [20], and for

$$
u(t)=0 \quad \forall t \in \mathbb{R}_{+}
$$


the system (1) is globally asymptotically stable at the origin for $|\beta|<\frac{k}{4}\left(\frac{\pi}{\ell}\right)^{2}$ [20] (the case of boundary conditions $z(t, 0)=z(t, \ell)=0$ for all $t \geq 0$ was studied in that monograph). The conclusion about global asymptotic stability in [20] is obtained using the LaSalle invariance principle and a Lyapunov function with a semi-definite time derivative calculated with respect to (1), which is the energy or the Hamiltonian of the undamped (i.e. $\alpha=0$ ) system:

$$
\mathcal{H}(\zeta)=\int_{0}^{\ell} \frac{z_{t}^{2}}{2}+k \frac{z_{x}^{2}}{2}+\beta(\operatorname{sign} \beta+\cos z) d x,
$$

then for $\mathcal{H}(t)=\mathcal{H}(\zeta(t, x))$ we have:

$$
\begin{aligned}
\dot{\mathcal{H}}(t)=\frac{d \mathcal{H}(t)}{d t} & =k z_{t}(t, \ell) u_{2}(t)+\int_{0}^{\ell} z_{t} u_{1}(t) d x \\
& -\alpha \int_{0}^{\ell} z_{t}^{2}(t, x) d x,
\end{aligned}
$$

that for (2) by the invariance principle implies convergence of the trajectories to the set where $z_{t}(t, x)=0$ for all $t \geq 0$, hence, to the equilibria.

For the system (1) under the restriction (2), the steady-state solutions $\xi \in H_{0}^{1}(I, \mathbb{R})$ have to satisfy the following partial differential equation:

$$
\xi_{x x}(x)=-\frac{\beta}{k} \sin \xi(x), x \in I
$$

together with the chosen boundary conditions. Actually, this system is well-investigated (e.g. as a nonlinear pendulum) and for $\beta>0$ the solutions are closed orbits for sufficiently small initial conditions, and for $\beta<0$ the unbounded curves are generated out the origin. Taking into account the boundary constraint in (1), (2):

$$
\xi_{x}(\ell)=0,
$$

implies that for $\ell<\frac{\pi}{2} \sqrt{\frac{k}{\beta}}$ the only possible solution of this equation (the only equilibrium) in our case is

$$
\xi(x)=0, x \in I,
$$

whose stability we are going to investigate (for $\beta \in \mathbb{R}$ ).

Our goal in this work is to propose a Lyapunov function for (1), which has a negative definite derivative under (2) and calculate a corresponding passive output signal (for the theory of passive and dissipative partial differential equations see [19], [1]). Next, we are going to develop some ISS conditions for (1) with respect to the input $u$ in the sense of [4], [13].

\section{STRICT PASSIVITY}

Consider the following candidate of a Lyapunov function:

$$
\begin{aligned}
V(\zeta)= & \int_{0}^{\ell}\left[\frac{z_{t}^{2}}{2}+a \frac{z_{x}^{2}}{2}+b \frac{\left(z_{t}+\alpha z\right)^{2}}{2}+c \frac{\left(z_{t}-\frac{\beta}{\alpha} \sin z\right)^{2}}{2}\right. \\
& +g(\operatorname{sign} \beta+\cos z)+e(1+\cos 2 z)] d x-f
\end{aligned}
$$

where $a>0, b>0, c>0, e>0$ and $g \in \mathbb{R}$ are parameters to select, $f=(g(\operatorname{sign} \beta+1)+2 e) \ell$. Let us check positive definiteness of this function $V(\zeta)$ for $\zeta \in \mathcal{Z}(I, \mathbb{R})$ (i.e. that $V(\zeta)>0$ for all $z \neq 0, z \in H_{0}^{1}(I, \mathbb{R})$ and $z_{t} \neq 0, z_{t} \in$ $L^{2}(I, \mathbb{R})$. Obviously, if a constraint

$$
\beta g>0
$$

is imposed, then $V(0)=0$. It is worth stressing that for $\zeta \in H^{1}(I, \mathbb{R}) \times L^{2}(I, \mathbb{R})$ it is not positive definite. But for $\zeta \in \mathcal{Z}(I, \mathbb{R})$ we obtain:

$$
\begin{aligned}
V(\zeta) \geq & \int_{0}^{\ell}\left[\frac{z_{t}^{2}}{2}+\frac{a}{2} z_{x}^{2}+g(\operatorname{sign} \beta+\cos z)\right. \\
& +e(1+\cos 2 z)] d x-f
\end{aligned}
$$

and recalling the inequality derived from the first term of Taylor expansion for $\cos z$ function:

$$
\cos z \geq 1-\frac{z^{2}}{2}
$$

the last inequality can be rewritten in the following way:

$$
\begin{aligned}
& V(\zeta) \geq \int_{0}^{\ell} \frac{z_{t}^{2}}{2}+\frac{a}{2} z_{x}^{2}+e\left(2-2 z^{2}\right) \\
& +g\left\{\begin{array}{ll}
2-\frac{z^{2}}{2} & \beta>0 \\
0 & \beta \leq 0
\end{array} d x-f\right. \\
& =\frac{1}{2} \int_{0}^{\ell} z_{t}^{2}+a z_{x}^{2}-\varrho z^{2} d x
\end{aligned}
$$

where

$$
\varrho=4 e+\left\{\begin{array}{ll}
g & \beta>0 \\
0 & \beta \leq 0
\end{array} .\right.
$$

And, finally, using Lemma 1 we get

$$
V(\zeta) \geq \frac{1}{2} \int_{0}^{\ell} z_{t}^{2}+\left(a-\varrho \frac{4 \ell^{2}}{\pi^{2}}\right) z_{x}^{2} d x .
$$

Therefore, a sufficient condition for the function $V(\zeta)$ to be positive definite can be written as follows:

$$
a \frac{\pi^{2}}{4 \ell^{2}}>\varrho
$$

Let us calculate the time derivative of $V(t)=V(\zeta(t, x))$ along the dynamics of (1):

$$
\begin{aligned}
\dot{V}= & \int_{0}^{\ell}\left[z_{t} z_{t t}+a z_{x} z_{x t}+b\left(z_{t}+\alpha z\right)\left(z_{t t}+\alpha z_{t}\right)\right. \\
& +c\left(z_{t}-\frac{\beta}{\alpha} \sin z\right)\left(z_{t t}-\frac{\beta}{\alpha} \cos z z_{t}\right)-g \sin z z_{t} \\
& \left.-2 e \sin 2 z z_{t}\right] d x \\
= & \int_{0}^{\ell}\left[z_{t}\left(k z_{x x}-\alpha z_{t}+\beta \sin z+u_{1}\right)+a z_{x} z_{x t}\right. \\
& +b\left(z_{t}+\alpha z\right)\left(k z_{x x}+\beta \sin z+u_{1}\right) \\
& +c\left(z_{t}-\frac{\beta}{\alpha} \sin z\right)\left(k z_{x x}-\alpha z_{t}+\beta \sin z+u_{1}\right. \\
& \left.\left.-\frac{\beta}{\alpha} \cos z z_{t}\right)-g \sin z z_{t}-2 e \sin 2 z z_{t}\right] d x .
\end{aligned}
$$


Then arranging the same terms we obtain:

$$
\begin{aligned}
\dot{V}= & \int_{0}^{\ell}\left[k(1+c+b) z_{x x} z_{t}-\left(\alpha+\frac{\beta}{\alpha} c \cos z\right) z_{t}^{2}+a z_{x} z_{x t}\right. \\
& +\alpha b k z_{x x} z+\alpha b \beta \sin z z \\
& -\alpha c\left(z_{t}-\frac{\beta}{\alpha} \sin z\right)^{2}-c k \frac{\beta}{\alpha} \sin z z_{x x} \\
& +\left(z_{t}+b\left(z_{t}+\alpha z\right)+c\left(z_{t}-\frac{\beta}{\alpha} \sin z\right)\right) u_{1} \\
& +(\beta(1+b)-g) \sin z z_{t} \\
& \left.+\left(\frac{c}{2}\left(\frac{\beta}{\alpha}\right)^{2}-2 e\right) \sin 2 z z_{t}\right] d x,
\end{aligned}
$$

and substituting the expressions from Proposition 1 the following estimate is derived:

$$
\begin{aligned}
\dot{V}= & k\left[(1+c+b) z_{t}(t, \ell)+\alpha b z(t, \ell)\right. \\
& \left.-c \frac{\beta}{\alpha} \sin z(t, \ell)\right] u_{2}(t) \\
& +\int_{0}^{\ell}\left[-\left(\alpha(1+c)+\frac{\beta}{\alpha} c \cos z\right) z_{t}^{2}-c \frac{\beta^{2}}{\alpha} \sin ^{2} z\right. \\
& -k\left(\alpha b-c \frac{\beta}{\alpha} \cos z\right) z_{x}^{2}+\alpha b \beta z \sin z \\
& +(a-k(1+c+b)) z_{x} z_{x t} \\
& +\left(z_{t}+b\left(z_{t}+\alpha z\right)+c\left(z_{t}-\frac{\beta}{\alpha} \sin z\right)\right) u_{1} \\
& +(\beta(1+b+2 c)-g) \sin z z_{t} \\
& \left.+\left(\frac{c}{2}\left(\frac{\beta}{\alpha}\right)^{2}-2 e\right) \sin 2 z z_{t}\right] d x .
\end{aligned}
$$

Denote

$$
\begin{aligned}
y_{1}(t)= & \int_{0}^{\ell}\left[(1+b+c) z_{t}+\alpha b z-c \frac{\beta}{\alpha} \sin z\right] d x \\
y_{2}(t)= & k\left[(1+c+b) z_{t}(t, \ell)+\alpha b z(t, \ell)\right. \\
& \left.-c \frac{\beta}{\alpha} \sin z(t, \ell)\right]
\end{aligned}
$$

as an auxiliary output $y \in C^{0}\left(\mathbb{R}_{+}, \mathbb{R}^{2}\right)$ of the system (1), select

$$
\begin{gathered}
a=k(1+c+b), \\
g=\beta(1+b+2 c), e=\frac{c}{4}\left(\frac{\beta}{\alpha}\right)^{2}
\end{gathered}
$$

and assume that

$$
\alpha+\left(\alpha-\frac{|\beta|}{\alpha}\right) c=\nu, \alpha b=c \frac{|\beta|}{\alpha}+\mu
$$

for some $\nu>0$ and $\mu>0$. The latter inequalities can be always ensured by a choice of $c>0$ and $b>0$ for some positive $\nu$ and $\mu$. In addition, note that the condition $\beta g>0$ is always satisfied for the proposed choice of the value of $g$, and (3) can be guaranteed for a sufficiently small values of $b>0$ and $c>0$ (which implies the positive definiteness of $V)$. Finally, we obtain:

$$
\begin{aligned}
\dot{V} \leq & \int_{0}^{\ell}\left[-\nu z_{t}^{2}-c \frac{\beta^{2}}{\alpha} \sin ^{2} z-k \mu z_{x}^{2}+\alpha b \beta z \sin z\right] d x \\
& +y^{\top}(t) u(t)
\end{aligned}
$$

and using Wirtinger's inequality from Lemma 1 we get:

$$
\dot{V} \leq \int_{0}^{\ell}\left[-\nu z_{t}^{2}-\frac{1}{2}\left[\begin{array}{c}
z \\
\sin z
\end{array}\right]^{\top} Q\left[\begin{array}{c}
z \\
\sin z
\end{array}\right]\right] d x+y^{\top}(t) u(t) \text {, }
$$

where

$$
Q=\left[\begin{array}{cc}
\mu \frac{k \pi^{2}}{2 \ell^{2}} & -\alpha b \beta \\
-\alpha b \beta & 2 c \frac{\beta^{2}}{\alpha}
\end{array}\right]
$$

The matrix $Q$ is positive definite if

$$
\frac{c}{\alpha} \mu \frac{k \pi^{2}}{\ell^{2}}>(\alpha b)^{2}
$$

then substituting the expression for $\alpha b$ we get

$$
\frac{c}{\alpha} \mu \frac{k \pi^{2}}{\ell^{2}}>\left(c \frac{|\beta|}{\alpha}+\mu\right)^{2}
$$

or equivalently (by performing straightforward computations)

$$
0>c^{2} \frac{\beta^{2}}{\alpha^{2}}+\frac{c}{\alpha}\left(2|\beta|-\frac{k \pi^{2}}{\ell^{2}}\right) \mu+\mu^{2} .
$$

Let us check that the latter inequality has a solution with respect to $\mu>0$. The roots of this quadratic equation are

$$
\mu_{1,2}=\frac{c}{2 \alpha} \frac{\sqrt{k} \pi}{\ell}\left(\frac{\sqrt{k} \pi}{\ell}-2|\beta| \pm \sqrt{\frac{k \pi^{2}}{\ell^{2}}-4|\beta|}\right)
$$

and a real solution exists if

$$
|\beta|<\frac{k}{4}\left(\frac{\pi}{\ell}\right)^{2}
$$

that corresponds to the conditions of global asymptotic stability of the equilibrium at the origin from [20] $\left(\frac{\sqrt{k} \pi}{\ell}-2|\beta|>0\right.$ under this restriction, then $\mu_{2}$ is always positive and the sign of $\mu_{1} \leq \mu_{2}$ is unimportant).

It is worth noting that the function $V(\zeta)+f$ is nonnegative by construction for all $\zeta \in \mathcal{Z}(I, \mathbb{R})$ under the imposed condition $\beta g>0$. Since for the restriction (8) the system (1) is globally asymptotically stable [20], $V(0)=0$ and $\dot{V}<0$ for all $\zeta(t) \neq 0$, then $V(\zeta)$ has to be positive definite and the condition (3) can be skipped from analysis (we cannot say that it is always satisfied since it is only sufficient for positive definiteness of $V(\zeta)$ ), but anyway as we explained above, (3) can be ensured by the choice of parameters $b$ and c.

Therefore, the following result has been proven:

Theorem 2. Let for the system (1) the restriction (8) be satisfied, then there exist $\mu>0$ and $\nu>0$ such that for parameters $a, b, c, g$,e validating the conditions (5) and (6), $V(\zeta)$ is a positive definite storage function for all $\zeta \in \mathcal{Z}(I, \mathbb{R}), u \in L^{\infty}\left(\mathbb{R}_{+}, \mathbb{R}^{2}\right)$ with the passive output 
$y \in C^{0}\left(\mathbb{R}_{+}, \mathbb{R}^{2}\right)$ given in (4), and it has a negative definite derivative in the case (2).

The same conclusion can be obtained using another Lyapunov function:

$$
\begin{aligned}
V(\zeta)= & \int_{0}^{\ell} \frac{z_{t}^{2}}{2}+a \frac{z_{x}^{2}}{2}+b \frac{\left(z_{t}+\alpha z\right)^{2}}{2}+c \sin z z_{t} \\
& +g(\operatorname{sign} \beta+\cos z) d x
\end{aligned}
$$

for suitably defined parameters $a>0, b>0, c>0$ and $g \beta>0$, with a passive output having the same terms, but investigation of this variant of $V(\zeta)$ requires a more involved analysis (e.g. for positive definiteness) and it is omitted here for brevity.

\section{INPUT-TO-STATE STABILITY}

The main result of this work is as follows:

Theorem 3. Let for the system (1) the restriction (8) be satisfied and

$$
u(t)=\left[\begin{array}{c}
v_{1}(t) \\
-\theta y_{2}(t)+v_{2}(t)
\end{array}\right],
$$

where $\theta>0$ is a design parameter and $v \in L^{\infty}\left(\mathbb{R}_{+}, \mathbb{R}^{2}\right)$ is a new input. Then (1) is ISS with respect to the input $v$.

Proof. Following [4], Theorem 1 can be used to prove this result. Let us show that the Lyapunov function $V(\zeta)$ given in Theorem 2 is an ISS-Lyapunov function for the system (1), (9). As it has been proven in Theorem $2, V$ is differentiable, positive definite and there exist $\alpha_{1}, \alpha_{2} \in \mathcal{K}_{\infty}$ such that

$$
\alpha_{1}\left(\|\zeta\|_{\mathcal{Z}(I, \mathbb{R})}\right) \leq V(\zeta) \leq \alpha_{2}\left(\|\zeta\|_{\mathcal{Z}(I, \mathbb{R})}\right)
$$

for all $\zeta \in \mathcal{Z}(I, \mathbb{R})$. Note that under (8) there is a tuning constant $\varsigma \in(0,1)$ such that

$$
|\beta|<\varsigma \frac{k}{4}\left(\frac{\pi}{\ell}\right)^{2}
$$

Under the substitution of (9) in the equations (1) we obtain the following estimate for the derivative of $V$ :

$$
\begin{aligned}
\dot{V} \leq & \int_{0}^{\ell}-\nu z_{t}^{2}-\frac{1}{2}\left[\begin{array}{c}
z \\
\sin z
\end{array}\right]^{\top} Q_{\varsigma}\left[\begin{array}{c}
z \\
\sin z
\end{array}\right] \\
& -(1-\varsigma) k \mu z_{x}^{2} d x \\
& +y_{1}(t) v_{1}(t)-\theta\left|y_{2}(t)\right|^{2}+y_{2}(t) v_{2}(t) \\
= & \int_{0}^{\ell}-\nu z_{t}^{2}-\frac{1}{2}\left[\begin{array}{c}
z \\
\sin z
\end{array}\right]^{\top} Q_{\varsigma}\left[\begin{array}{c}
z \\
\sin z
\end{array}\right] \\
& -(1-\varsigma) k \mu z_{x}^{2} d x \\
& +\left((1+b+c) z_{t}+\alpha b z-c \frac{\beta}{\alpha} \sin z\right) v_{1} d x \\
& -\theta y_{2}^{2}(t)+y_{2}(t) v_{2}(t),
\end{aligned}
$$

where

$$
Q_{\varsigma}=\left[\begin{array}{cc}
\varsigma \mu \frac{k \pi^{2}}{2 \ell^{2}} & -\alpha b \beta \\
-\alpha b \beta & 2 c \frac{\beta^{2}}{\alpha}
\end{array}\right] .
$$

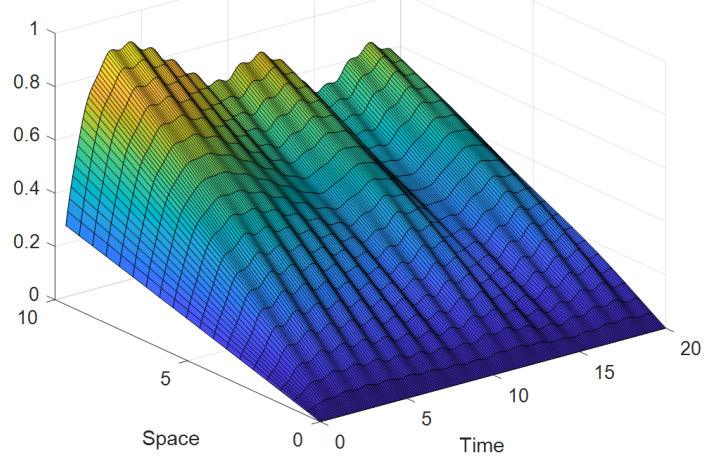

Figure 1. The plot of $z(t, x)$

Since under (8) the matrix $Q_{\varsigma}$ is positive definite (this property can be established similarly as in the proof of Theorem 2 for $Q$ ), then using Young's inequality (i.e. $a b \leq \frac{|a|^{p}}{p}+\frac{|b|^{q}}{q}$ for any $a, b \in \mathbb{R}$ and $p, q \in \mathbb{R}_{+}$with $\frac{1}{p}+\frac{1}{q}=1$ ) it is possible to show that there exist $\epsilon_{1}>0, \epsilon_{2}>0, \sigma_{1}>0$ and $\sigma_{2}>0$ such that

$$
\begin{aligned}
\dot{V} \leq & \int_{0}^{\ell}-\epsilon_{1} z_{t}^{2}-\epsilon_{2} z_{x}^{2} d x+\sigma_{1} v_{1}^{2}(t)+\sigma_{2} v_{2}^{2}(t) \\
\leq & -\min \left\{\epsilon_{1}, \epsilon_{2}\right\}\|\zeta\|_{\mathcal{Z}(I, \mathbb{R})} \\
& +\max \left\{\sigma_{1}, \sigma_{2}\right\}\|v\|_{L^{\infty}\left(\mathbb{R}_{+}, \mathbb{R}^{2}\right)},
\end{aligned}
$$

which is sufficient for the required ISS property by Theorem 1.

The main conclusions of Theorem 3 are that under (8), the ISS property of (1) can be established with a feedback transformation of the input (9) at the boundary.

\section{Simulations}

Let

$$
\begin{gathered}
\ell=10, k=1, \alpha=0.7, \beta=\varsigma \frac{k}{4} \frac{\pi^{2}}{\ell^{2}}, \varsigma=0.9, \\
u_{1}(t)=-0.2 \cos (5 t), u_{2}(t)=0.1 \sin (t) .
\end{gathered}
$$

For discretization in space and in time, the explicit Euler method is used with the space discretization step $\frac{\ell}{N-1}$ and the time discretization step 0.0002 , where $N=100$ is the number of points chosen on the interval $I$. The initial conditions are selected as

$$
z^{0}(x)=\sin \left(\frac{\pi}{2} \frac{x}{\ell}\right), z^{1}(x)=\sin \left(3 \frac{x}{\ell}\right)
$$

satisfying the boundary constraints. Then the results of simulation, $z(t, x)$ and $z_{t}(t, x)$, are shown in figures 1 and 2, respectively. They illustrate the theoretical findings of our work. 


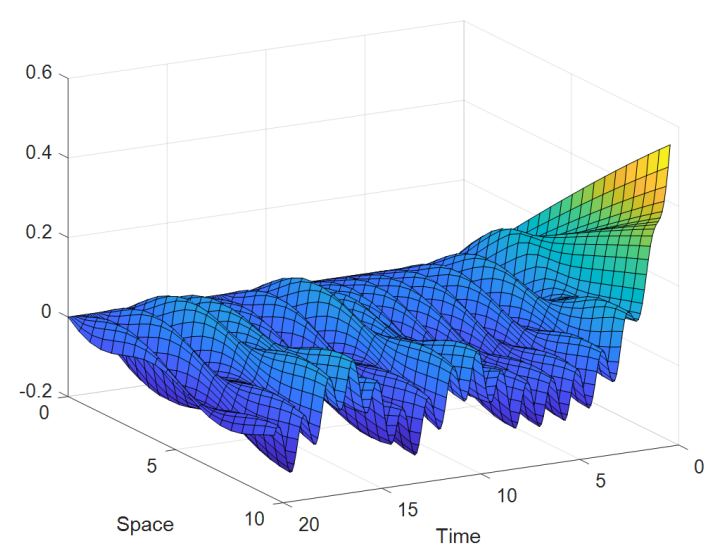

Figure 2. The plot of $z_{t}(t, x)$

\section{CONCLUSIONS}

The conditions of strict passivity and input-to-state stability are investigated for a sine-Gordon equation with perturbations introduced in the right-hand side of the model and in the boundary conditions. The case of a single equilibrium at the origin is considered and a strict Lyapunov function is designed for the noise-free case. It is discovered that sineGordon equation possesses robust stability with respect to the disturbances in the dynamics, but an additional damping has to be applied at the boundary to guarantee the same tolerance to the input entering there. Further directions of research may include analysis of other boundary conditions and the case with multiple equilibria.

\section{REFERENCES}

[1] A.A. Alonso, V.F. Carlos, and R.B. Julio. Dissipative systems: from physics to robust nonlinear control. International Journal of Robust and Nonlinear Control, 14(2):157-179, 2004.

[2] K. Bredies, C. Clason, K. Kunisch, and G. von Winckel. Control and optimization with PDE constraints, volume 164 of International Series of Numerical Mathematics. Birkhäuser, Basel, 2013.

[3] R. Curtain and H. Zwart. An introduction to infinite-dimensional linear systems theory. Springer-Verlag, New York, 1995.

[4] S. Dashkovskiy and A. Mironchenko. Input-to-state stability of infinitedimensional control systems. Mathematics of Control, Signals, and Systems, 25(1):1-35, 2013.

[5] S.N. Dashkovskiy, D.V. Efimov, and E.D. Sontag. Input to state stability and allied system properties. Automation and Remote Control, 72(8):1579, 2011.

[6] R.W. Dickey. Stability theory for the damped sine-Gordon equation. SIAM Journal on Applied Mathematics, 30(2):248-262, 1976.

[7] M. Dolgopolik, A. L. Fradkov, and B. Andrievsky. Boundary energy control of a system governed by the nonlinear Klein-Gordon equation. Math. Control Signals Syst., 30(3):1-7, 2018.

[8] M.V. Dolgopolik and A.L. Fradkov. Energy tracking for the sinegordon equation with dissipation via boundary control. In European Control Conference, pages 3025-3030, Limassol, 2018.

[9] A. Friedman. Partial differential equations of parabolic type. PrenticeHall, Inc., Englewood Cliffs, N.J., 1964.

[10] G.H. Hardy, J.E. Littlewood, and G. Polya. Inequalities. Cambridge University Press, 1988.

[11] T. Kobayashi. Boundary feedback stabilization of the sine-Gordon equation without velocity feedback. Journal of Sound and Vibration, 266:775-784, 2003.
[12] I. Lasiecka and R. Triggiani. Control Theory for Partial Differential Equations, volume 1 of Abstract Parabolic Systems: Continuous and Approximation Theories. Cambridge University Press, 2000.

[13] A. Mironchenko and F. Wirth. Characterizations of input-to-state stability for infinite-dimensional systems. IEEE Transactions on Automatic Control, 63(6):1692-1707, 2018.

[14] A. Pazy. Semigroups of linear operators and applications to partial differential equations. Springer, 1983.

[15] A.V. Porubov, A.L. Fradkov, and B.R. Andrievsky. Feedback control for some solutions of the sine-Gordon equation. Appl. Math. Comput., 269(C):17-22, 2015.

[16] A. Smyshlyaev and M. Krstic. Adaptive Control of Parabolic PDEs. Princeton University Press, 2010.

[17] R. Temam. Infinite-Dimensional Dynamical Systems in Mechanics and Physics. Springer, New York, 1997.

[18] M. Tucsnak and G. Weiss. Observation and control for operator semigroups. Birkhäuser, Basel, 2009.

[19] A.J. van der Schaft and B.M. Maschke. Hamiltonian formulation of distributed-parameter systems with boundary energy flow. Journal of Geometry and Physics, 42(1):166-194, 2002.

[20] J.A. Walker. Dynamical Systems and Evolution Equations, Theory and Application. Plenum Press, New York, 1980. 